\title{
"Implementation of a palliative care service in a university hospital. Challenges and perspectives"
}

\begin{abstract}
The Palliative Care philosophy and practice remains largely unknown in Brazil. This lack of knowledge substantially impacts in the quality of medical care for terminal patients. This article describes the implementation of a Palliative Care Service in a University Hospital, where the needs, motivations, achievements and difficulties faced were described. In Western medicine there is a professional training deficiency in understanding the process of dying. Therefore, it is important to include the Palliative Care teaching in graduation, as well as to disseminate knowledge to physicians in training. The implementation of a Palliative Care Service in a University Hospital comes up as a great opportunity to link medical assistence, teaching and research.
\end{abstract}

Keywords: palliative care, service implementation, medical education
Volume 2 Issue 4 - 2018

\author{
Josenília M.A. Gomes, Claudia Regina \\ Fernandes, Liane Carvalho de Brito de Souza, \\ Cinara Franco de Sá Nascimento Abreu, \\ Luciana Leite de Figueiredo Magalhães, \\ Manuela Vasconcelos de Castro Sales \\ Walter CantÃ $d i o$ University Hospital, CapitÃ fo Francisco \\ Pedro
}

Correspondence: Josenília M.A. Gomes, Walter Cant $\tilde{A}\urcorner d i o$ University Hospital, Capit ̃̃fo Francisco Pedro St, I290, Fax +55 992563323,Email gomes.josenilia@gmail.com

Received: February 26, 2018 | Published: August 28, 2018

\begin{abstract}
Abbreviations: PPS, palliative performance scale; HUWC, Walter cantídio university hospital; EBSERH, Brazilian hospital services company; ICU, intensive care unit; CFM, Council of Medicine
\end{abstract}

\section{Introduction}

Palliative care is a multidisciplinary approach foccus on improving the patients and families quality of life through prevention and relief of suffering based on early identification and precise control of symptoms as well as the treatment of pain and other physical, psychological or spiritual problems while they facing a life threatening illness.Efficient palliative care services should be universally available and integrated at all levels of health care, such as general hospitals, emergencies, rehabilitation centers, long-term institutions, community programs and home care. ${ }^{1}$

Attention to patients in a terminal situation becomes more important each day, due to the aging population and the increase of patients with chronic diseases. The technological advance in the health area has been contributing to increase the life expectancy of patients with chronic diseases, who are generally fragile and have many functional limitations, requiring greater medical and care support in order to provide them a better quality and dignity of life. As a result, the increase in health spending can be observed, either in the form of disease-modifying treatments, clinical intercurrences or rehabilitation, overloading families and the health system. Most of these patients will be hospitalized and undergo treatments that will not change the natural history of the disease. By the other hand, this may cause more suffering for the patient and distress for the families. ${ }^{2}$

Dignity and quality of life should undoubtedly be the maingoal of treatment in these patients. "Helping to end life with dignity and respecting the intrinsic value of each person is surely a greater way of exercising medicine and health care in general, which must be encouraged and developed". The clinical decision should never be separate from its basis and ethical aspects. Each decision making should take the evolution parameters of the disease, the severity of the current crisis, the frequency of complications, the patient opinion (if possible), the opinion of medical staff and family, the symptom control and the comfort measures. The patient should not be a victim of abandonment. All suitable measures of comfort should be taken (beneficence), and nothing that could exacerbates or prolongs the disease suffering stage (not maleficence) should be done, not insisting on the maintenance of a patient whose terminal characteristics of the disease must be understood. ${ }^{3}$

In Brazil there is still a great unfamiliarity about the Palliative Care practice among physicians, health system managers, health professionals, judiciary system and population. Many people remain confused with euthanasia. There is a huge damage associated with a lack of adequate training for opioids use to control symptoms. This gap in training physicians and health professionals in Palliative Care is due to the absence of medical residency programs and quality specialization courses. ${ }^{4}$

The implementation of the first Palliative Care services in Brazil is very recent, and emerged in the late 1990s. Even today, most services are focused on the care of patients with cancer and on the pain management. ${ }^{5}$

There are several challenges to be overcame in order to implement Palliative Care in health institutions. We can take as exemple absence of national policy for pain relief, deficiency in health and community education, concerns about the use of morphine and other opioids, limitations on the supply of other drugs for the relief of pain, deficiency in the training of physicians, lack of financial resources for research and development in palliative care. ${ }^{6}$

The implantation of Palliative Care Services in educational institutions such as University Hospitals is imperative in the current global health scenario. It is the opportunity to combine teaching and assistance ensuring greater dissemination of knowledge to professionals during their training, as well as providing a field for training graduation and postgraduate students.

\section{Method}

This article aims to describe the implementation of a Palliative Care Service in a University Hospital. It is therefore a preliminary report of the motivations, achievements and difficulties faced during 
this process. Walter Cantídio University Hospital(HUWC) is a high complexity hospital where teaching and research activities of the Faculty of Medicine of the Federal University of Ceará and other health schools are developed. It is also a training ground for medical and multidisciplinary residents in several specialities. It stands out for the care of low-income people, chronic patients, seriously ill and with multiple comorbidities in clinical and surgical specialities. It is a reference hospital for bone marrow and solid organs transplantations, with a large number of such procedures. Since 2013 it is managed by the Brazilian Hospital Services Company (EBSERH).

\section{Results}

The HUWC Palliative Care Service started its activities in March 2016, after public selection and the recruitment of three physicians trained in Palliative Medicin and one medical coordinator. The other participants of the team consisted of a nurse trained in palliative care, a psychologist and a social worker. Thus, the service team was composed of four doctors and a nurse, all of them with exclusive hours for the service; a psychologist, partially assigned by the Department of Cardiology and a social worker, partially assigned by the Hemodialysis service, what meens that they were not exclusive of the Palliative Care Service.

This is a consulting team, which is request according to the perception of the attending physician of the patient in the form of a written opinion. The team does not conduct of the patient alone, but guides or suggests tratment options that should be analyzed by the attending physician and may or may not be implemented.

Clinical Cases are discussed in daily manner. In addition, there is a weekly meeting with the interdisciplinary team, where all members participate and share the difficulties, seeking solutions to more complex cases. Scientifics updates are also held at these meetings with classes and discussions of scientific articles.

The staff is housed in a room, with a secretariat that receives and distributes the requests of consultation. In this place, family and weekly meetings are held with the interdisciplinary team. The advantage of this way of team working is that it functions as a rapid disseminator of the philosophy of end-of-life care, as it is in frequent contact with a large amount of hospital staff, contributing to the educating role of care workers. The disadvantage is having to work with unprepared staff who may have difficulty accepting some aspects of palliative care promptly, requiring some time for this adaptation. ${ }^{7}$

In addition to responding to the new requests, the team also makes frequent visits to follow the evolution of the patients, as well as modify conducts or therapeutic plans when necessary. Family meetings are held together with the assistant team, assisting in the process of physician-patient-family communication.

There is also an outpatient clinic in palliative care where patients who have been discharged from hospital as well as other patients referred from the other services that fit the care profile of chronic life threatening patients continued ambulatorial assistance.

Nurses make daily visits to patients and family members, evaluate functionality/prognosis through the Palliative Performance Scale (PPS) and symptom control through the Edmont Symptom Scale, as well as performing a nursing assessment and adjusting care to the best comfort of the patient. The nursing perspective is well focused on important issues such as wounds/ostomies, drug delivery routes (hypodermoclisis), appropriate use of opioid rescue doses. The daily follow-up of the palliative nurses establishes an important bridge of communication with the unit nursing team and the family that is of fundamental importance for the good progress of the care for the family, reinforcing the objectives and the philosophy of palliative care. The nurse also sinalize the doctor for a case of greater urgency of intervention.

Psychologist follows the cases of patients and families with greater emotional distress and difficulties in dealing with the illness and acceptance of death. Provides systematic psychological care to hospitalized patients and their families. It also carries out outpatient psychological care, including assistance to bereaved. When necessary, some patients/family are referred to the psychiatry service.

The Social Service makes a careful socioeconomic evaluation through a social interview identifying the social support network, detecting the demands according to the patients' needs, such as benefitsand referrals to the for the acquisition of medicine and materials. The articulation with the support network is of great importance for the continuity of the treatment at home and during the care of the family/caregivers are verified the necessary conditions for social discharge.

In the first few months, HUWC's palliative care team was sparked. The consultancies were scarce and related to cancer patients at the end of life, patients of the Intensive Care Unit (ICU) and communication of difficult news.

A common misunderstanding at this stage was that the function of the palliative care team was "persuading" the family to discontinue nutritional, ventilatory, hemodynamic support in a clear confusion between palliative care and euthanasia, and revealing a profound lack of understanding of the basic principles guiding and conduct the palliative care assistance.

This scenario motivated the team to invest in training. The palliative care team actively participated in the clinical sessions of some services to discuss clinical cases, as well as promoted classes with basic notions for medical residents of Internal Medicine.

The result of this educational process was the increase in the number of consultation requests as well as a greater qualification of these, as there was an increase in requests to follow up chronic non-oncological clinical diseases, neurodegenerative diseases, hematological and infectious diseases, as well as management of symptom control. There was also an increase in the interest of the residents to discuss cases and participate in the service during some clinical rotations.

In addition to residentes training, contact with the undergraduate medical students was also started through an internship in anesthesiology. Weekly, interns participate in the team's activities, giving opinions, attending family meetings and in outpatient care. In addition to discussing the cases in a weekly meeting with an interdisciplinary team.

\section{Discussion}

The medicalization of death in Western society is reflected in the treatment of the terminally ill patient and immediately refers to hospital care, because in this culture, those who are very sick seek a hospital. Therefore, it is in hospitals where the main palliative care services in Brazil appear.?

There are several models of palliative care assistance: hospital, home and outpatient. In the hospital model, care can be performed in 
three ways: palliative care unit; advisory team and itinerant team. The palliative care unit consists of a set of beds where a trained staff works under the palliative philosophy. In this situation the team works more cohesively and the goals are reached with greater ease. The advisory team works in the form of opinions and runs the entire hospital, giving suggestions and guiding behaviors. The intinerantee team is also triggered by the attending physician, but assumes the bed. This may exclude the assisting staff from getting involved in patients' end-oflife care. ${ }^{7}$ This makes it clear that there is no single, ideal model for care delivery, which should be determined on the basis of local needs and resources.

According to data from the Global Alliance for Palliative Care more than 100 million people benefit from palliative care annually (including family members and caregivers), yet represent less than $8 \%$ who need this type of care and have their access actually guaranteed. This scenario most likely occurs because training in palliative care is rarely included in the educational curriculum of health professionals. In addition, the availability of pain drugs - the most basic topic when it comes to minimizing patient suffering - is fully inadequate in most parts of the world, often because of concerns about its illicit use and drug trafficking. These data point to the need for training of all health professionals to provide basic palliative measures, called palliative actions, thus configuring the university hospital as a place where a palliative care service is essential.

In Western Medicine there is a deficiency in training of health professionals in the sense of understanding the process of death and dying. The professional sees the death of his patient as a failure, an enemy. ${ }^{9}$ For this reason, the inclusion of Palliative Care teaching in undergraduate courses promotes a revision in the way of understanding death that is now seen as a natural process, part of life.

In practice, what is often observed is a profound lack of knowledge on the part of physicians and other health professionals about the indications and therapeutic goal of palliative care, which is the quality of life of patients with life-threatening illness and their family. Ignorance results in prejudice and fear of legal consequences related to therapeutic limitations.

Therefore, the resolutions of the Brazilian Council of Medicine (CFM) related to Palliative Care, as well as some cases of welldocumented jurisprudence are always reinforced in the classes and discussions of continued education in the HUWC, as well as with undergraduate students who pass through service.

Some countries already have specific standards and legislation for this care implemented. In France, a law was passed (99477 of June 9, 1999) which guarantees the right of terminally ill patients to access palliative care, rescuing the protection and dignity of the human person. This law also improves the training of health professionals in this area and encourages the formation of volunteers. ${ }^{6}$

In Brazil, the CFM included in its code of ethics, resolution 1805/2006, which in its article 1 says: "The physician is allowed to limit or suspend procedures and treatments that prolong the life of the patient in the terminal stage, of a serious and incurable disease, respected the will of the person and his legal representative ". Article 2 highlights the need for continued care in the form of palliative care: "The patient will continue to receive all the necessary care to alleviate the symptoms that lead to suffering, ensuring complete care, physical, psychic, social and spiritual comfort, including assuring him the right to discharge himself". ${ }^{10}$
With the objective of promoting the humanization of care, public policies have been stimulating the creation of centers specialized in palliative care. In Brazil, Portaria n 1.319/GM, of July 2002, creates for the SUS the centers of reference in the treatment of Pain, which in many hospitals are the embryos for the implantation of services in Palliative Care. ${ }^{6}$

Despite the existence of these public policies, the Death Quality Index indicates Brazil occupying $30^{\circ}$ place in 2010 and $420^{\circ}$ place in 2015. This index is produced by the Economist Intelligence Unit, commissioned by the Lien Foundation-a philanthropic organization of Singapore and represents a quantitative and qualitative analysis, assessing ethical and social aspects related to the dying process, involving four categories related to end-of-life care: environment health care, availability of care, costs and quality. That is, there is much that still needs to be done to effectively produce improvements in the dying process in Brazil. Using the classification proposed by the WHO to categorize countries according to the prevalence of palliative care services they present, Brazil is in category $3 \mathrm{a}$ - isolated provision. In other words, few services are concentrated in small areas of the country. This gap needs to be addressed and this can only occur with the implementation of more palliative care services, particularly in university hospitals where care and education co-exist satisfactorily by forming potential leaders of other future services. ${ }^{11}$

Many difficulties were identified over the months during the implementation of the service, highlighting the lack of knowledge regarding the indications and benefits of Palliative Care in patients / family of patients with life threatening illness. This difficulty has been overcome with the permanent education of all those involved in the care, but must be maintained especially considering the reality of a university hospital constant renovation of the medical residents and other health professions from schools where palliative care does not exist as curricular content.

Another difficulty that needs to be overcome is the resizing of the multiprofessional team, since the predominant profile of HUWC patients is chronic patients with life-threatening advanced diseases, and therefore many of them are eligible for palliative care and, thus with dissemination of the knowledge of the correct indications will increase demand for intervention of the consulting team that if, remaining of the current size, will be in the near future, insufficient to adequately respond to the growing number of requests.

In addition to the initial challenges and difficulties inherent in the implementation of a new service, it must be remembered at this point, all the resitences related to the important cultural innovations palliative care philosophy brings up. Cleary there are, two very strong paradigms within of the academic environment peculiar to a traditional university hospital with more than 60years of existence that have to be overcome: the way of dealing with the death process and the unreasonable use of health technology.

Finally the secret to keep moving towards is in the empathy and compassion for the patients and their families, constant reflection of practice, permanent education, evaluation of the results and the team mutual support.

\section{Conclusion}

Implementing a medical care service in a University Hospital for patients suffering from life-threatening diseases, especially in terminality, allows deeping the discussions of these issues throghout the academic rounds. 
One important challenge is to secure as many as possible oportunities for total undergraduate students and residents of all clinical and surgical specialities to go through the service, living daily activities, supporting patients and families to improve their palliative care knowledge. In addition, it is important to maintain a continuing education schedule that encompasses multiprofessional teams including the residents of other areas of health and the hospital stuff itself.

HUWC Palliative Care team needs in the present scenario, exclusive professionals of all areas, as well as increase the number of nurses in the service and inclusion of other professionals such as physiotherapists, occupational therapist and nutritionist among others. Due to the hospital's profile, creating a specific Palliative Care Unit would also be very necessary, as well as extending this service to deliver home care, of course, improving quality and attention to families. In addition to promoting a better distribution of the hospital beds according to the needs.

As a very navy service it still doesn't have enough comparative date against other older services to evidence the impact on quality of care and the economy, but despite the short time of operation, the pallitive care team already has benefited and promoted improvements in the quality of life and death in the HUWC and this experience proves that even without large amounts of money is possible to change reality making it better for people who suffers.

\section{Acknowledgements}

None.

\section{Conflict of interest}

The author declares no conflict of interest.

\section{References}

1. Moraes N. et al. Cuidados Paliativos com Enfoque Geriátrico - A Assistência Multidisciplinar. São Paulo: Editora Atheneu; 2014

2. Durillo FTP. Montalbán ANG, Purificación Jaén Castillo, et al. Análisis de la atividade de un equipo de suporte de cuidados paliativos. Medicina Paliativa. 2011;18(4):129-134

3. Rosinhas JM. Limitações Terapêuticas Em Indivíduos Portadores De Demência Avançada. Instituto De Ciências Biomédicas De Abel SalazarUniversidade Do Porto; 2012. p. 1-38

4. ANCP. ANCP e Cuidados Paliativos no Brasil. ANCP; 2017.

5. Palmeira Heloísa Maria, Scorsolini-Comin Fabio, Peres Rodrigo Sanches. Cuidados paliativos no Brasil: revisão integrativa da literatura científica. Aletheia. 2011;35-36:179-189

6. Roncarati R, de Camargo RMP, Rossetto EG, et.al. Cuidados paliativos num hospital Universitário de Assistência Terciária: uma necessidade? Semina Ciências Biológicas E da Saúde. 2003;24(1):37-48.

7. Bifulco VA, Iochida LC. A formação na graduação dos profissionais de saúde e a educação para o cuidado de pacientes fora de recursos terapêuticos de cura. Rev bras educ med. 2009;33(1):92-100.

8. Conselho federal de medicina. Resoluções/CFM. 2006;1:6.

9. Manual de cuidados Paliativos ANCP. Academia Nacional de Cuidados Paliativos; 2012. p. 11-585.

10. Global atlas on end of life care disponívelem the worldwide hospice palliative care alliance; 2018

11. Thomas Lynch T, Connor S, Clark D. Mapping levels of palliative care development: a global update. J Pain Symptom Manage. 2013;45(6):1094-1106. 